\title{
GENERIC DEFORMATIONS OF LAGRANGIAN AND LEGENDRIAN MAPS
}

\author{
SERGEY STANCHENKO \\ Moscow Aviation Institute \\ Volokolamskoe shosse, 4, 125871 Moscow, Russia \\ E-mail: stan@k804.mainet.msk.su
}

\section{Introduction}

a. Investigating ocean and atmosphere flows Nye and Thorndike [2] have studied typical bifurcations of three dimensional vector fields depending on time. One can describe such a field as a one-parameter family of maps from $R^{3}$ to $R^{3}$ or as a map from $R^{1} \times R^{3}=$ $R^{4}$ to $R^{3}$.

To study them the authors of [2] consider sections of stable maps from $R^{4}$ to $R^{4}$. There is one family in their list of typical sections for which the set of critical values for an isolated value of the parameter is equal to the caustic of a Lagrangian $D_{4}$ map.

This leads to another problem: To study properties of Lagrangian and Legendrian maps included in generic families of maps with a suitable number of parameters. In this way $V$-versal deformations of Lagrangian $D_{k}$ and Legendrian $A_{k}$ maps are considered below.

b. As a Lagrangian map is the restriction to a Lagrangian submanifold of the projection that defines a Lagrangian fiber bundle, Lagrangian maps may be locally considered as maps from $R^{n}$ to $R^{n}$. The normal form of Lagrangian stable maps is given by the corresponding classification theorem $([1])$.

ExAmple. The normal form of Lagrangian $A_{k}$ maps coincides with that of stable Whitney maps $A_{k}$ :

$$
\begin{gathered}
A_{k}:\left(R^{n}(x), 0\right) \rightarrow\left(R^{n}(y), 0\right), \\
y_{1}= \pm(k+1) x_{1}^{k}+(k-1) x_{2} x_{1}^{k-2}+\ldots+2 x_{k-1} x_{1}, \\
y_{i}=x_{i}, \quad i=2, \ldots, n, \quad k-1 \leq n
\end{gathered}
$$

1991 Mathematics Subject Classification: Primary 58C27; Secondary 53C15. Research supported by ISF grant MSD000 and by RFFI grant 94-01-00255.

The paper is in final form and no version of it will be published elsewhere. 
It is obvious that Lagrangian $A_{k}$ maps are stable in the class of general maps.

EXAMPLE. The Lagrangian $D_{k}^{ \pm}$maps have the following normal form:

$$
\begin{aligned}
& \quad D_{k}^{ \pm}:\left(R^{n}(x), 0\right) \rightarrow\left(R^{n}(y), 0\right), \\
& y_{1}=x_{2}^{2} \pm(k-1) x_{1}^{k-2}+(k-2) x_{3} x_{1}^{k-3}+\ldots+2 x_{k-1} x_{1}, \\
& y_{2}=2 x_{1} x_{2}, \\
& y_{i}=x_{i} \quad i=3, \ldots, n, \quad k-1 \leq n, \quad k \geq 4 .
\end{aligned}
$$

In this paper the properties of the maps (2) are described. The $V$-versal deformation preserving the origin is a one parameter deformation for the maps (2). For even $k$ the Lagrangian $D_{k}^{+}$maps fall into stable maps $A_{k}$ (at two isolated points), the Lagrangian $D_{k}^{-}$maps decompose only into $A_{k-1}, A_{k-2}$ etc. For odd $k$ the Lagrangian $D_{k}$ maps fall into $A_{k}$ (at one isolated point).

c. A map from a Legendrian submanifold to the base of a Legendrian bundle may be locally considered as a map from $R^{n}$ to $R^{n+1}$.

EXAmPLE. The normal form of Legendrian $A_{k}$ maps is given by (see [1])

$$
L A_{k}:\left(R^{n}(y, x), 0\right) \rightarrow\left(R^{n+1}(q), 0\right),
$$

$$
\begin{aligned}
& q_{1}=\varphi_{1}(y, x), \\
& q_{i}=x_{i}, \quad i=2, \ldots, n, \quad q_{n+1}=\varphi_{2}(y, x),
\end{aligned}
$$

where

$$
\begin{aligned}
& \varphi_{1}=(k+1) y^{k}+(k-1) x_{2} y^{k-2}+\ldots+2 x_{k-1} y, \\
& \varphi_{2}=k x^{k+1}+(k-2) x_{2} x_{1}^{k-1}+\ldots+x_{k-1} y^{2} .
\end{aligned}
$$

In the Legendrian case the following results are obtained: The $V$-versal deformation of Lagrangian $A_{k}$ maps preserving the origin is a $k-1$-parameter deformation. The bifurcational diagram for this family is constructed. Outside the bifurcational set the maps of this family are stable and at isolated points they are $R L$-equivalent to the trivial extension of the stable maps that has the image of a "Whitney umbrella". Legendrian $A_{k}$ maps have infinite $R L$ - and topological codimension.

The author is grateful to Prof. V. Zakalyukin for his considerate attention to this work.

\section{2. $V$-versal deformation of Lagrangian $D_{k}$ maps}

Proposition 1. The $V$-versal deformation $D_{k}^{ \pm}(t, c)$ of the maps (2) is given by

$$
\begin{aligned}
& y_{1}=\varphi_{1}(x)+t x_{2}+c_{1}, \\
& y_{2}=\varphi_{2}(x)+c_{2}, \\
& y_{i}=x_{i}+c_{i}, \quad i=3, \ldots, n,
\end{aligned}
$$

where

$$
\begin{aligned}
& \varphi_{1}(x)=x_{2}^{2} \pm(k-1) x_{1}^{k-2}+(k-2) x_{3} x_{1}^{k-3}+\ldots+2 x_{k-1} x_{1} \\
& \varphi_{2}(x)=2 x_{1} x_{2}
\end{aligned}
$$


Pr o of. Direct calculations.

The main result for Lagrangian $D_{k}$ series. Let $D_{k}(t)$ be the family (4) with $c=0: D_{k}^{ \pm}(t)=D_{k}^{ \pm}(t, 0)$, and let $k-1=n$.

THEOREM 1. If $k$ is even, $t \neq 0$, then $D_{k}^{+}(t)$ has 2 singular points at which it is $R L$-equivalent to $A_{k}(1)$. These points have coordinates

$$
x_{10}= \pm s_{1}|t|^{2 /(k-2)}, \quad x_{20}=-t / k, \quad x_{i 0}=s_{i} x_{10}^{i-2}, \quad i=3, \ldots, n .
$$

$D_{k}^{-}(t)$ has no $A_{k}$ points.

If $k$ is odd, $t \neq 0$, then $D_{k}(t)$ has one singular point at which it is $R L$-equivalent to $A_{k}$. This point has coordinates

$$
x_{10}=s_{1} t^{2 /(k-2)}, \quad x_{20}=-t / k, \quad x_{i 0}=s_{i} x_{10}^{i-2}, \quad i=3, \ldots, n,
$$

for some $s_{1}, s_{3}, \ldots, s_{n}$.

Proof. It is sufficient to prove two propositions:

A. If $t \neq 0$, then $D_{k}^{ \pm}(t)$ has the corresponding number of singular points with Boardman type $\Sigma^{\frac{1 \ldots 1}{k}}$.

B. $D_{k}^{ \pm}(t)$ is stable at these points.

First we find all the points of $\Sigma^{\frac{1 \ldots 1}{k}}$ Boardman type.

LEMMA 1. Let $x_{1}^{2}+x_{2}^{2} \neq 0$. Then for $D_{k}^{ \pm}(t)$

$$
\Sigma^{\frac{1 \ldots 1}{i}}=\left\{x \in R^{n} \mid B_{1}(x)=0, \ldots B_{i-1}(x)=0, B_{i}(x) \neq 0\right\},
$$

where

$$
B_{i}=b_{i 1} x_{1}^{k-2}+b_{i 2} x_{3} x_{1}^{k-3}+\ldots+b_{i k-2} x_{k-1} x_{1}+b_{i k-1} x_{2}+b_{i k} x_{2}^{2}
$$

and $\left(b_{i j}\right)=B$ is the $(k-1) \times k$-matrix:

(5)

$$
\left(\begin{array}{cccccc} 
\pm(k-1)(k-2) & (k-2)(k-3) & \ldots & 2 & -t & -2 \\
\mp(k-1)(k-2)^{2} & -(k-2)(k-3)^{2} & \ldots & -2 & -t & -4 \\
\ldots & \ldots & & & & \\
(-1)^{k-2}(k-1)(k-2)^{k-1} & (-1)^{k-2}(k-2)(k-3)^{k-1} & \ldots & (-1)^{k-2} 2 & -t & -2^{k-1}
\end{array}\right)
$$

Pr o of. Direct calculations.

Let $S=\left\{\left(x_{1}, \ldots, x_{n}\right) \in R^{n} \mid x_{1}=0, x_{2}=0\right\}$.

LEMma 2. $S$ contains no points of $\Sigma^{\frac{1 \ldots 10}{k}}$ Boardman type, and it contains one point of $\Sigma^{\frac{1 \ldots 1}{k-1}}$ Boardman type. This point is $(0 \ldots 0)$.

Thus to find the points of $\Sigma^{\frac{1 \ldots 1}{k-1}}$ Boardman type we should solve the system of equations

$$
B_{1}=0, \ldots, B_{k-1}=0
$$

where $B_{i}$ are as in lemma 1 . This is a system of linear algebraic equations over the 
monomials $x_{1}^{k-2}, x_{3} x_{1}^{k-3}, \ldots, x_{k-1} x_{1}, x_{2}, x_{2}^{2}$. It may be represented in the following way:

$$
\left(\tilde{b}_{i j}\right) \cdot\left(\begin{array}{c}
x_{1}^{k-2} \\
x_{3} x_{1}^{k-3} \\
\vdots \\
x_{k-1} x_{1} \\
x_{2}
\end{array}\right)=x_{2}^{2} \cdot\left(\begin{array}{c}
2 \\
4 \\
\vdots \\
2^{k-1}
\end{array}\right),
$$

where $\left(\tilde{b}_{i j}\right)$ is the matrix $\left(b_{i j}\right)$ without the last column. If the linear system with $(k-1) \times(k-1)$ matrix $\left(\tilde{b}_{i j}\right)$ is solvable, then for some values $s_{1}, \ldots, s_{k-1}$,

$$
x_{1}^{k-1}=s_{1} x_{2}^{2}, \quad t x_{2}=s_{2} x_{2}^{2}, \quad x_{i} x_{1}^{k-i}=s_{i} x_{2}^{2}, \quad i=3, \ldots, k-1 .
$$

The equation $t x_{2}=s_{2} x_{2}^{2}$ has 2 solutions: $x_{20}=t / s_{2}$ and $x_{20}=0$. The second solution is non-proper by lemma 2. Then the number of solutions of the system (6) is equal to that of the equation $x_{1}^{k-2}=s_{1} x_{2}^{2}$.

If $k$ is odd, then this equation has one real solution, and $D_{k}(t)$ has one point of $\sum^{\frac{1 \ldots 1}{k-1}}$ type. To complete the proof of proposition A we need the following algebraic lemma:

LEMMA 3. Let $M=\left(m_{i j}\right)$ be a $k \times n$ matrix $(n>k)$ with each column a geometric progression with ratio $l_{i}, l_{i} \neq l_{j}$. Then there exists a non-singular $k \times k$ matrix $C$ such that $C \cdot M$ is as follows:

$$
\left(\begin{array}{ccccc}
* & & & \ldots & \\
0 & \ddots & & \ldots & \\
& 0 & m_{k k} q_{k} & \ldots & m_{k n} q_{n}
\end{array}\right)
$$

where

$$
q_{i}=\left(1-l_{1} / l_{i}\right) \ldots\left(1-l_{k-1} / l_{i}\right)
$$

In other words we can see the elements of the last row of $M$ after reducing it to the triangle matrix.

Pr o of. Direct calculations.

Using Lemma 3 we may get the following results:

COROllary 1. If $x_{0}=\left(x_{01} \ldots x_{0 k-1}\right)$ is the solution of the system (6) and $x_{02} \neq 0$ then

$$
\pm x_{01}^{k-2}=\frac{(-1)^{k-2}}{k-1} \cdot\left(\frac{t}{k}\right)^{2}, \quad x_{02}=-\frac{t}{k} .
$$

Corollary 2. If $k$ is even, then $D_{k}(t)$ has two points of $\sum^{\frac{1 \ldots 1}{k-1}}$ type and for these points

$$
x_{01}=\frac{ \pm 1}{1-k}\left(\frac{t}{k}\right)^{\frac{2}{k-2}}, \quad x_{02}=-\frac{t}{k} .
$$

If $k$ is odd, then $D_{k}(t)$ has one point of $\sum^{\frac{1 \ldots 1}{k-1}}$ type, and

$$
x_{01}=\frac{1}{1-k}\left(\frac{t}{k}\right)^{\frac{2}{k-2}}, \quad x_{02}=-\frac{t}{k} .
$$


Corollary 3. If $x_{0}$ is a point of $\sum^{\frac{1 \ldots 1}{k-1}}$ Boardman type for $D_{k}(t)$, then $B_{k}\left(x_{0}\right) \neq 0$ (i.e. $x_{0}$ is a point of $\Sigma^{\frac{1 \ldots .1}{k}}$ Boardman type for $D_{k}(t)$ ).

That completes the proof of proposition A.

Corollary 4. 1) $B_{1} \ldots B_{k-1} \in \mathbf{m}\left(x_{1}-x_{01}, \ldots, x_{k-1}-x_{0 k-1}\right)$.

2) $\left|\frac{\partial\left(B_{1}, \ldots, B_{k-2}\right)}{\partial\left(x_{2}, \ldots, x_{k-1}\right)}\right|_{x=x_{0}} \neq 0$.

Now to prove the stability of $D_{k}(t)$ at $x_{0}$ we use the following construction: Let the germ of $D:\left(R^{n}, x_{0}\right) \rightarrow\left(R^{n}, y_{0}\right)$ have the Boardman type $\Sigma^{\frac{1 \ldots 10}{k}}$ at $x_{0}$, and $\eta$ be the germ of a smooth vector field whose direction coincides with the direction of the null-space of the derivative of the map $D$. Consider the functions $B_{i}(x)$ such that $B_{1}(x)$ is the Jacobian of $D$,

$$
B_{2}(x)=d B_{1}(\eta), \ldots, B_{k}=d B_{k-1}(\eta) .
$$

Obviously, $B_{1}\left(x_{0}\right)=\ldots=B_{k-1}\left(x_{0}\right)=0$.

Proposition 2. If the differentials $d B_{1} \ldots d B_{k-2}$ are independent at $x_{0}$, then the germ $D$ is $R L$-equivalent to the germ of a Whitney $A_{k}$ map at $x_{0}$.

By corollary 4 if $D_{k}(t)$ has the Boardman type $\Sigma^{\frac{1 \ldots 10}{k}}$ at $x_{0}$, then all the conditions of proposition 2 are fulfilled. That completes the proof of the theorem.

3. Proof of proposition 2. This proof is based on two simple lemmas.

Lemma 4. In some coordinates $u$ and $v$ the germ of $D$ may be represented by

$$
\begin{gathered}
D:\left(R^{n}(u), 0\right) \rightarrow\left(R^{n}(v), 0\right), \\
v_{1}=\varphi(u), \quad v_{i}=u_{i}, \quad i=2, \ldots, k-1,
\end{gathered}
$$

where

$$
\begin{gathered}
\varphi(u)=u_{1}^{k}+\varphi_{1}\left(u_{2} \ldots u_{n}\right) u_{1}^{k-1}+\ldots+\varphi_{k-1}\left(u_{2} \ldots u_{n}\right) u_{1}+\varphi_{k} \\
\varphi_{1}, \ldots, \varphi_{k-1} \in \mathbf{m}(u), \quad n=k-1,
\end{gathered}
$$

and in these coordinates $\eta=\partial / \partial u_{1}$.

LEMMA 5. The following conditions are equivalent:

1) The germ of map (8) is stable at 0.

2)

3)

$$
\left|\frac{\partial\left(\varphi_{2}, \ldots, \varphi_{k-1}\right)}{\partial\left(u_{2}, \ldots, u_{k-1}\right)}\right|_{u=0} \neq 0
$$

$$
\left|\frac{\partial\left(\varphi^{\prime}, \ldots, \varphi^{(k-2)}\right)}{\partial\left(u_{2}, \ldots, u_{k-1}\right)}\right|_{u=0} \neq 0
$$

where $\varphi^{(i)}=\partial^{i} \varphi / \partial u_{1}^{i}$.

4)

$$
\left|\frac{\partial\left(B_{1}, \ldots, B_{k-2}\right)}{\partial\left(x_{2}, \ldots, x_{k-1}\right)}\right|_{x=x_{0}} \neq 0
$$


where the basis vectors of the coordinates $x_{2}, \ldots, x_{k-1}$ are transversal to the vector $\eta$ at $x_{0}$.

Proof of lemma 5 . a) 1) $\Leftrightarrow 2$ ). This follows from the theorem on stability of expansion of genotype [1].

b) 2$) \Leftrightarrow 3$ ). This follows from the rules of differentiation.

c) The functions $B_{1}, \ldots, B_{k-2}$ are the sequential derivatives in the direction of $\eta$ of the Jacobian $B_{1}=|\partial y / \partial x|$. The functions $\varphi^{\prime}, \ldots, \varphi^{(k-2)}$ are the sequential derivatives in the direction of $\eta$ of the Jacobian $K=|\partial y / \partial x|$.

Thus the ideals generated by $B_{1}, \ldots, B_{k-2}$ and by $\varphi^{\prime}, \ldots, \varphi^{(k-2)}$ coincide. The basis vectors of the coordinates $x_{2}, \ldots, x_{k-1}$ are transversal to the vector $\eta$, and the coordinates $u_{2}, \ldots, u_{k-1}$ have the same property. Then 3) and 4) are equivalent.

\section{4. $V$-versal deformations of Legendrian $A_{k}$ maps}

Proposition 3. The $V$-versal deformation of the map (3) is given by

$$
\begin{aligned}
q_{1} & =\varphi(y, x)+c_{1}, \\
q_{i} & =x_{i}+c_{i}, \quad i=2, \ldots, n, \\
q_{n+1} & =\varphi_{2}(\lambda, y)+P(\lambda, y)+c_{n+1},
\end{aligned}
$$

where $y \in R^{1}, x \in R^{n-1}, \lambda \in R^{k-1}, c \in R^{n+1}$ and

$$
\begin{aligned}
\varphi_{1} & =(k+1) y^{k}+(k-1) x_{2} y^{k-1}+\ldots+2 x_{k-1} y, \\
\varphi_{2} & =k y^{k+1}+(k-2) x_{2} y^{k-1}+\ldots+x_{k-1} y^{2}, \\
P(\lambda, y) & =\lambda_{1} y^{k-1}+\ldots+\lambda_{k-2} y^{2}+\lambda_{k-1} y .
\end{aligned}
$$

Let $\Sigma_{P} \subset R^{k-1}(\lambda)$ be the discriminant set for the polynomial $P^{\prime}(\lambda, y)=\partial P / \partial y$.

THEOREM 2. If $\lambda \in \Sigma_{P}$ then the maps (9) are nonstable for each c. If $\lambda \notin \Sigma_{P}$ then the maps (9) are stable. Their image is RL-equivalent to the trivial extension of the "Whitney umbrella". The preimage of the umbrellas set is a finite combination of planes of codimension 2.

Proof. The Jacobi matrix is

$$
\left(\begin{array}{cc}
\varphi_{1}^{\prime} & * \\
0 & E_{n-2} \\
y \varphi_{1}^{\prime}+P^{\prime} & * *
\end{array}\right)
$$

where $\varphi^{\prime}=\partial \varphi / \partial y, P^{\prime}=\partial P / \partial y$.

The vector field $\eta=\partial / \partial y$ coincides with the direction of the null-space of $M$. The set $\Sigma^{\frac{1 \ldots 1}{l}}=\Sigma^{1_{l}}$ is defined by the equations

$$
\begin{array}{llll}
\varphi_{1}^{\prime}(y, x)=0, & \varphi_{1}^{\prime \prime}(y, x)=0, & & \varphi_{1}^{(l)}(y, x)=0 \\
P^{\prime}(\lambda, y)=0, & P^{\prime \prime}(\lambda, y)=0, & & P^{(l)}(\lambda, y)=0 .
\end{array}
$$

If $y_{0}$ is a root of $P^{\prime}(\lambda, y)=0$ with multiplicity $l$, then $\Sigma^{1_{l}}$ is defined by $l+1$ equations

$$
y=y_{0}, \quad \varphi^{\prime}\left(y_{0}, x\right)=0, \ldots, \varphi^{(l)}\left(y_{0}, x\right)=0 .
$$


Thus $\Sigma^{1_{l}}$ is a plane of codimension $l+1$. According to the Boardman formula this codimension is $2 l$. Then if $l>1$, we have nonstability. In case $l=1$ after some calculations, we may see the extension of the "Whitney umbrella".

Corollary 5. The $V$-versal deformation of a Legendrian $A_{3}$ map is a 2-parameter deformation. It consists of maps equivalent to the "umbrella" at not more than one point.

5. Generic deformations of Legendrian $A_{k}$ maps. Now we compare $V$ - and $R L$-equivalence for deformations of Legandrian $A_{k}$ maps. It is easy to prove

Proposition 4. A generic deformation of a Legendrian $A_{k}$ map $(k \geq 3)$ is $R L$ equivalent to the following deformation:

$$
\begin{aligned}
q_{1} & =\varphi_{1}, \\
q_{i} & =x_{i}, \quad i=2, \ldots, n, \\
q_{n+1} & =\varphi_{2}+h(x, y),
\end{aligned}
$$

where $\varphi_{1}$ and $\varphi_{2}$ are the same as in $(3)$, and $h(x, y)$ is an arbitrary smooth function.

As was shown in the preceding section the $V$-versal deformation of the Legendrian $A_{3}$ map has not more than one "umbrella" point. Another situation is for generic deformations:

Let $O_{\epsilon}$ be the $\epsilon$-sphere in the space of all coefficients of the Taylor series of $h$ at $0, Q_{\delta}$ be the $\delta$-sphere around the origin in $R^{3}(q)$ and let $n=2$.

Proposition 5. For arbitrary $\epsilon>0, \delta>0$, and integer $m$ there is a function $h$ such that

1) All the Taylor coefficients of $h$ are in $O_{\epsilon}$.

2) The map (10) is equivalent to the "Whitney umbrella" at $m$ points and all the preimages of these points are in $Q_{\delta}$.

Proof. All the points at which the image of the map (10) is equivalent to the "umbrella" can be defined from the system of equations

$$
\varphi_{1 y}^{\prime}=0, \quad h_{y}^{\prime}=0 .
$$

We can take the polynomial $h=h_{1}+h_{2} y+\ldots+h_{m} y^{m}$ with sufficiently small coefficients.

Thus Legendrian $A_{k}$ maps have no finite $R L$-versal and finite topologically versal deformations.

\section{References}

[1] V. I. Arnold, S. M. Gusein-Zade and A. N. Varchenko, Singularities of Differentiable Maps, Vol. 1, Nauka, Moscow, 1982 (in Russian); English transl.: Birkhäuser, 1985.

[2] J. F. Nye and A. S. Thorndike, Events in evolving three-dimensional vector fields, J. Phys. A Math. Gen. 13 (1980), 1-14. 